\title{
The Relativistic Stern-Gerlach Interaction and Quantum Mechanics Implications *
}

\author{
P. Cameron*, M. Conte ${ }^{\dagger}$, A. U. Luccio*, W. W. MacKay*, M. Palazzi ${ }^{\dagger}$ and \\ M. Pusterla** \\ * Brookhaven National Laboratory, Upton, NY 11973, USA. \\ ${ }^{\dagger}$ Dipartimento di Fisica dell'Università di Genova, INFN Sezione di Genova, Via Dodecaneso 33, \\ 16146 Genova, Italy. \\ ** Dipartimento di Fisica dell'Università di Padova, INFN Sezione di Padova, Via Marzolo 8 , \\ 35131 Padova, Italy.
}

\begin{abstract}
The time varying relativistic Stern-Gerlach force, which acts over a charged particle endowed with a magnetic moment, is deduced from the Dirac Hamiltonian finding its coincidence with the classical expression. Possible drawbacks related to the Heisenberg uncertainty principle are discussed.
\end{abstract}

\section{THE FORMALISM OF THE STERN-GERLACH INTERACTION}

The effect of the Stern-Gerlach (SG) force over a charged particle endowed with a magnetic moment $\vec{\mu}^{*}$ has been evaluated mainly in the perspective of achieving the separation of the two polarization states of fermion beams, either by acting on the trajectory slopes[1][2] while crossing constant magnetic gradient, or by exploiting the energy differences [3][4] related to the interaction between magnetic moments and electromagnetic fields of a suitable if cavity. The latter system has also been proposed [5] as a polarimeter. In the particle rest frame (PRF), where all the quantities are labelled with a prime, this force takes the expression

$$
\vec{f}_{S G}^{\prime}=\nabla^{\prime}\left(\vec{\mu}^{*} \cdot \vec{B}^{\prime}\right)=\frac{\partial}{\partial x^{\prime}}\left(\vec{\mu}^{*} \cdot \vec{B}^{\prime}\right) \hat{x}+\frac{\partial}{\partial y^{\prime}}\left(\vec{\mu}^{*} \cdot \vec{B}^{\prime}\right) \hat{y}+\frac{\partial}{\partial z^{\prime}}\left(\vec{\mu}^{*} \cdot \vec{B}^{\prime}\right) \hat{z},
$$

which when boosted back to the laboratory frame (LAB) becomes

$$
\vec{f}_{S G}=\frac{1}{\gamma} \frac{\partial}{\partial x}\left(\vec{\mu}^{*} \cdot \vec{B}^{\prime}\right) \hat{x}+\frac{1}{\gamma} \frac{\partial}{\partial y}\left(\vec{\mu}^{*} \cdot \vec{B}^{\prime}\right) \hat{y}+\frac{\partial}{\partial z^{\prime}}\left(\vec{\mu}^{*} \cdot \vec{B}^{\prime}\right) \hat{z},
$$

where $\beta$ and $\gamma$ are the usual relativistic parameters, and the $z$-axis has been chosen parallel to the particle velocity $\vec{v}$. Bearing in mind the relation between $\vec{B}^{\prime}$ and the fields $\vec{B}, \vec{E}$ in the laboratory frame, the longitudinal component of the SG force (2) becomes

$$
\begin{aligned}
f_{z} & =\mu_{x}^{*} C_{z x}+\mu_{y}^{*} C_{z y}+\mu_{z}^{*} C_{z z}, \quad \text { with } \\
C_{z x} & =\gamma^{2}\left[\left(\frac{\partial B_{x}}{\partial z}+\frac{\beta}{c} \frac{\partial B_{x}}{\partial t}\right)+\frac{\beta}{c}\left(\frac{\partial E_{y}}{\partial z}+\frac{\beta}{c} \frac{\partial E_{y}}{\partial t}\right)\right], \\
C_{z y} & =\gamma^{2}\left[\left(\frac{\partial B_{y}}{\partial z}+\frac{\beta}{c} \frac{\partial B_{y}}{\partial t}\right)-\frac{\beta}{c}\left(\frac{\partial E_{x}}{\partial z}+\frac{\beta}{c} \frac{\partial E_{x}}{\partial t}\right)\right],
\end{aligned}
$$

CP675, Spin 2002: 15th Int'l. Spin Physics Symposium and Workshop on Polarized Electron Sources and Polarimeters, edited by Y. I. Makdisi, A. U. Luccio, and W. W. MacKay (C) 2003 American Institute of Physics 0-7354-0136-5/03/\$20.00 


$$
C_{z z}=\gamma\left(\frac{\partial B_{z}}{\partial z}+\frac{\beta}{c} \frac{\partial B_{z}}{\partial t}\right) \text {. }
$$

It is relevant to point out that Eq. (3) can be deduced from the quantum relativistic theory of the spin- $\frac{1}{2}$ charged particle (Dirac). Indeed, if we start from the Hamiltonian

$$
H=e \phi+c \vec{\alpha} \cdot(\vec{p}-e \vec{A})+\gamma_{0} m c^{2},
$$

with the Dirac's matrices

$$
\vec{\gamma}=\left(\begin{array}{cc}
0 & \vec{\sigma} \\
-\vec{\sigma} & 0
\end{array}\right), \quad \gamma_{0}=\left(\begin{array}{cc}
I_{2} & 0 \\
0 & -I_{2}
\end{array}\right), \quad \vec{\alpha}=\gamma_{0} \vec{\gamma}=\left(\begin{array}{cc}
0 & \vec{\sigma} \\
\vec{\sigma} & 0
\end{array}\right)
$$

where $\vec{\sigma}$ is a vector whose components are the Pauli's matrices

$$
\sigma_{x}=\left(\begin{array}{cc}
0 & -i \\
i & 0
\end{array}\right), \quad \sigma_{y}=\left(\begin{array}{cc}
1 & 0 \\
0 & -1
\end{array}\right), \quad \sigma_{z}=\left(\begin{array}{ll}
0 & 1 \\
1 & 0
\end{array}\right), \quad \text { and } \quad I_{2}=\left(\begin{array}{ll}
1 & 0 \\
0 & 1
\end{array}\right)
$$

Here we have chosen the $y$-axis as the one parallel to the main magnetic field. By means of standard steps it is straightforward to derive the nonrelativistic expression of the Hamiltonian exhibiting the SG interaction with the "normal" magnetic moment

$$
\tilde{H}=e \phi+\frac{1}{2 m}(\vec{p}-e \vec{A})^{2}-\frac{e \hbar}{2 m}(\vec{\sigma} \cdot \vec{B})
$$

which coincides with the Pauli equation. In particular this equation is valid in the PRF.

At this stage we must add to the Stern-Gerlach energy the contribution from the anomalous magnetic moment, which gives rise to a factor $1+a=g / 2$, thus obtaining

$$
-\frac{g}{2} \frac{e \hbar}{2 m} \vec{\sigma} \cdot \vec{B}=-\vec{\mu}^{*} \cdot \vec{B} \quad \text { with } \quad \vec{\mu}^{*}=g \frac{e \hbar}{4 m} \vec{\sigma} .
$$

In order to obtain the $z$-component of the SG force in the Laboratory frame, we must boost the whole Pauli term of Eq. (10) by using the unitary operator $U[6]$ in the Hilbert space, which expresses the Lorentz transformation:

$$
U^{-1}\left[g \frac{e \hbar}{4 m}\left(\gamma_{0} \vec{\sigma} \cdot \vec{B}^{\prime}\right)\right] U=g \frac{e \hbar}{4 m} S^{-1}\left(\gamma_{0} \sigma_{x}\right) S B_{x}+S^{-1}\left(\gamma_{0} \sigma_{y}\right) S B_{y}+S^{-1}\left(\gamma_{0} \sigma_{z}\right) S B_{z}
$$

where

$$
\begin{gathered}
S=\exp \left[\gamma_{0}(\vec{\gamma} \cdot \hat{v}) \frac{u}{2}\right]=I_{4} \cosh \frac{u}{2}+\left(\begin{array}{cc}
0 & \sigma_{z} \\
\sigma_{z} & 0
\end{array}\right) \sinh \frac{u}{2}, \\
\hat{v}=\frac{\vec{v}}{|\vec{v}|}, \quad \cosh u=\frac{1}{\sqrt{1-\beta^{2}}}=\gamma \quad \text { (Lorentz factor), and } \beta=\frac{v}{c} .
\end{gathered}
$$

Here $I_{4}$ is the $4 \times 4$ identity matrix. From the algebraic properties of the $\gamma$ and $\sigma$ matrices, we obtain (extending the $\vec{\sigma}$ to $4 \times 4$ matrices with the $2 \times 2$ Pauli matrices repeated in the diagonal blocks)

$$
\begin{aligned}
& S^{-1}\left(\gamma_{0} \sigma_{x}\right) S=\gamma_{0} \sigma_{x}, \\
& S^{-1}\left(\gamma_{0} \sigma_{y}\right) S=\gamma_{0} \sigma_{y}, \quad \text { and }
\end{aligned}
$$




$$
\begin{aligned}
S^{-1}\left(\gamma_{0} \sigma_{z}\right) S & =\gamma\left(\gamma_{0} \sigma_{z}\right)+i \sqrt{\gamma^{2}-1} \gamma_{0} \gamma_{5}, \quad \text { with } \\
\gamma_{5} & =\gamma_{x} \gamma_{y} \gamma_{z} \gamma_{0}=i\left(\begin{array}{cc}
0 & I_{2} \\
I_{2} & 0
\end{array}\right) .
\end{aligned}
$$

At this stage it is straightforward to deduce the expectation values of the SG force in the LAB system with a defined spin component (along the $y$-axis in our case) via the expectation values of the Pauli interaction term and Lorentz transformation of the proper force:

$$
f_{z}=\gamma^{2} \mu^{*}\left[\left(\frac{\partial B_{y}}{\partial z}+\frac{\beta}{c} \frac{\partial B_{y}}{\partial t}\right)-\frac{\beta}{c}\left(\frac{\partial E_{x}}{\partial z}+\frac{\beta}{c} \frac{\partial E_{x}}{\partial t}\right)\right] .
$$

In fact, only Eq. (16) gives a non null result, while both Eqs. (15) and (17) yield a null contribution to the expectation values quoted above, because of orthogonality of the two spin states $s= \pm \frac{1}{2}$ and the properties of the $\sigma$ matrices.

It should be noted that the electric field $\vec{E}$ and the time derivative appearing in Eq. (19) are due to the well known Lorentz transformations

$$
\begin{aligned}
\vec{E}^{\prime} & =\gamma(\vec{E}+c \vec{\beta} \times \vec{B})-\frac{\gamma^{2}}{\gamma+1}, \vec{\beta}(\vec{\beta} \cdot \vec{E}) \\
\vec{B}^{\prime} & =\gamma\left(\vec{B}-\frac{\vec{\beta}}{c} \times \vec{E}\right)-\frac{\gamma^{2}}{\gamma+1} \vec{\beta}(\vec{\beta} \cdot \vec{B}), \\
\frac{\partial}{\partial x^{\prime}} & =\frac{\partial}{\partial x}, \quad \frac{\partial}{\partial y^{\prime}}=\frac{\partial}{\partial y}, \quad \text { and } \quad \frac{\partial}{\partial z^{\prime}}=\gamma\left(\frac{\partial}{\partial z}+\frac{\beta}{c} \frac{\partial}{\partial t}\right) .
\end{aligned}
$$

It is interesting to underline that Eq. (19) coincides with the Eq. (3) if we keep in mind that only a single spin component can be measured.

\section{QUANTUM LIMITATIONS}

Let us now consider the effect of a static quadrupole field $\vec{B}=(G y, G x, 0)$ on the trajectory slopes. Particles with spins parallel to the $y$-axis undergo a horizontal impulse, or kick, $\delta p_{x}=\mu^{*} G \ell_{Q} / \beta c\left(\ell_{Q}=\right.$ quadrupole length $)$ which generates two slope variations

$$
\eta_{ \pm}=\frac{\delta p_{x}}{p}= \pm \frac{\mu^{*} G \ell_{Q}}{\beta^{2} \gamma m c^{2}} \quad(p=\text { particle momentum })
$$

Hence particles with opposite spin states will have, on the long run, separate trajectories. Eq. (23) shows the method is not viable at high energy since $|\eta|$ fades off with the growth of $\gamma$.

On the contrary, in the case of the energy exchange between particles and the electromagnetic field of a rf cavity, both Eqs. (4) and (5) exhibit a factor $\gamma^{2}$ which is also present in the expression of the energy gained (or lost) during a cavity crossing. Let us recall the typical example [4] dealing with a rectangular cavity excited in its $\mathrm{TE}_{011}$ mode:

$$
\Delta U_{ \pm}= \pm 2 \gamma^{2} \mu^{*} B_{0}
$$


where $B_{0}$ is the cavity's peak magnetic field. The energy variation (24) makes the beam's momentum spread vary by the amount

$$
\left(\frac{\Delta p}{p}\right)_{ \pm}= \pm \frac{2 \gamma}{\beta^{2}} \frac{\mu^{*} B_{0}}{m c^{2}} \simeq \pm 2 \gamma \frac{\mu^{*} B_{0}}{m c^{2}} \quad \text { (ultrarelativistic) }
$$

which implies that the energy separation between particles with opposite spin states is more and more effective as the beam's energy increases.

We return to the case of the nonrelativistic transverse kick [7], considering the classical betatron oscillation of a particle, circulating in a ring, as the motion of the center of its quantum wave packet which represents [8],[9] the wave-function of the "corresponding state". Moreover, when the particle crosses a focusing quadrupole, we can treat its motion with the quantum harmonic oscillator thus obtaining the following expressions for the momentum and position uncertainties:

$$
\Delta x_{0}=\sqrt{\frac{\hbar}{\sqrt{m v e G}}} \quad \text { and } \quad \Delta p_{0}=\sqrt{\hbar \sqrt{m v e G}}
$$

As soon as the particle leaves the focusing region and travels along either a free space or a defocusing quadrupole, no discrete energy levels exist anymore and a pure continuous spectrum will appear; this implies a dilation of the wave-packet size, according to the relation

$$
\Delta x_{w p}=\Delta x_{0} \sqrt{1+\frac{\lambda^{2}}{\left(\Delta x_{0}\right)^{4}} \frac{z^{2}}{4 \beta^{2}}} \simeq \frac{\lambda}{2 \beta \Delta x_{0}} z
$$

where $z$ is the space covered by the particle outside the focusing quadrupole and $\lambda=$ $\hbar / m c$ is the Compton wave length of the particle.

On the other hand, the momentum uncertainty (26) generates an angular deflection $x_{q}^{\prime}=\frac{\Delta p_{0}}{m v}$ which gives rise to a spatial increment $\Delta x_{q}=\frac{\Delta p_{0}}{m v} z$ over a length $z$. Comparing this growth to the size (27) of the swollen wave-packet, we obtain:

$$
\frac{\Delta x_{q}}{\Delta x_{w p}}=\frac{\Delta p_{0}}{m v} z \frac{2 m v \Delta x_{0}}{\hbar i z}=\frac{2 \Delta p_{0} \Delta x_{0}}{\hbar}=2
$$

If we set, for instance, $G=10 \mathrm{Tm}^{-1}$ and $v=3.095 \times 10^{7} \mathrm{~ms}^{-1}$ or $\beta=0.103(5 \mathrm{MeV}$ protons), from Eq. (26) we obtain $\Delta x_{0}=1.93 \times 10^{-8}$, while for $z=10 \mathrm{~m} \mathrm{Eq.} \mathrm{(27)}$ yields $\Delta x_{w p}=5.37 \times 10^{-7} \mathrm{~m}$. With all these in mind, we may find, for $\ell=1 \mathrm{~m}$, $\delta p_{x}=4.56 \times 10^{-33} \mathrm{kgms}^{-1}$ or $\delta p_{x} \Delta x_{q} \simeq 10^{-39} \mathrm{Js} \ll \hbar \simeq 10^{-34}$ Js. However, the random nature of the quantum uncertainty does not allow its growth to macroscopic size; on the contrary, the coherence of our classical kicks permits overcoming the uncertainty limit and realizing a macroscopic effect by addition. In our example, the small spatial increments $\delta x=\left(\delta p_{x} / p\right) z=8.80 \times 10^{-13} \mathrm{~m}$ will sum up till reaching the value of $\Delta x_{q}$ after about $10^{6}$ revolutions.

As far as the energy gain/loss is concerned, the situation is simpler and more straightforward. In fact in the relativistic case, with $\Delta E$ from Eq. (24), $\Delta t$ being of size $\frac{1}{2} \tau_{\text {rf }} \simeq 10^{-10} \mathrm{~s}$ (i.e. a half period of $3 \mathrm{GHz}$ ) and $B_{0}=0.1 \mathrm{~T}$, we obtain:

$$
\Delta E \Delta t \simeq \begin{cases}\gamma^{2} 1.85 \times 10^{-34} \mathrm{Js} & \text { for electrons } \\ \gamma^{2} 2.82 \times 10^{-37} \mathrm{Js} & \text { for protons }\end{cases}
$$


From Eq. (29) it is easy to infer that, in the case of protons, the uncertainty principle constraints are overwhelmed with $\gamma \geq 19$ or $\gamma \geq 48.5$ depending whether we have chosen $\hbar$ or $h$ as limiting quantity. As far as electrons are concerned, even nonrelativistic particles skip these constraints.

We have demonstrated the possibility of detecting sizeable effects of the SG force over a free fermion. We have made it clear that, even in the example of the transverse impulse hidden in the uncertainty blur, this kick can repeat turn after turn giving thus rise to a measurable effect, provided that reasonable operating conditions are settled. Energy exchanges between the particle's kinetic energy and the cavity's electromagnetic fields can be detected with an efficiency proportional to $\gamma$.

\section{CONCLUDING REMARKS}

Under the hypothesis that the PRF is inertial we proved the equivalence of the classical and quantum mechanical relativistic SG force. We are aware that such an issue is an approximation because the actual motion of the test particle is neither straight nor uniform in any real accelerator. However, the time spent inside the cavities is much shorter than the revolution period and this fully justifies our approximation.

Another relevant application to consider is the proposal of constructing an absolute polarimeter. To be more specific, this might consist of a passive if cavity, placed along the beam axis, which should detect a total energy transfer of the order of

$$
\Delta U \simeq 2 N P \mu^{*} B_{0} \gamma^{2}
$$

where $N$ is the number of particles belonging to a bunch train, $P$ is the beam polarization and $B_{0}$ is now the self-field created by the crossing particles.

Finally, it should be noted that the continuous Stern-Gerlach effect has been recently observed for an electron in an atomic ion[10].

\section{REFERENCES}

1. T. Niinikoski and R. Rossmanith, Nucl. Instrum. Methods, A255, 460 (1987).

2. Y. Onel, A. Penzo and R. Rossmanith, AIP Conf. Proc., Vol. 150, 1229 (1986),

3. M. Conte, A. Penzo and M. Pusterla, Il Nuovo Cimento, A108 (1995) 127.

4. M. Conte et al., INFN/TC-00/03, (http:xxx.lanl.gov/listphysics/0003, preprint 0003069); M. Conte et al., ICFA Beam Dynamics Newsletter, 24, 66 (2001) (http://wwwslap.cern.ch/icfa/); P. Cameron et al., Proc. of the SPIN2000 Symposium, Osaka, AIP Conf. Proc. 570, 785 (2001).

5. P. Cameron et al., PAC2001, 2403 (2001).

6. R.P. Feynman, Quantum Electrodynamics, W.A. Benjamin Inc., New York 1961.

7. M. Conte and M. Pusterla, Il Nuovo Cimento, 103A, 1087 (1990).

8. L.I. Schiff, Quantum Mechanics, McGraw-Hill Co. Inc., New York 1949.

9. P. Caldirola, R. Cirelli e G.M. Prosperi, Introduzione alla Fisica Teorica, UTET, Torino 1982. (In Italian)

10. N. Hermanspahn et al., Phys. Rev. Lett. 84, 427 (2000). 